\title{
Los agronegocios y la innovación en la sociedad de la modernidad líquida.
}

\section{Agribusiness and innovation in the society of liquid modernity.}

\begin{abstract}
Como citar este artículo / To reference this articcle: Arosa-Carrera Charles R., (2016). Valoración de Empresa Transportadora en el Meta: Estudio de Caso EL CONUCO: (investigación, economía y sociedad), 3(2), pp. 1-9. DOI: https://doi.org/10.22579/2619-614X.769
\end{abstract}

"La cultura es concebida libre, no para no pertenecer, sino para pertenecer a todos." Arosa-Carrera Ch (2020).

Charles R. Arosa-Carrera ${ }^{1}$

\section{Artículo de investigación}

Recepción: 15-12-2020

Aceptación: 20-05-2021

El Conuco es una revista de acceso abierto revisada por pares. C $2018 \mathrm{El}$ autor (es). Este es un artículo de acceso abierto distribuido bajo los términos de la Licencia Internacional Creative Commons Attribution 4.0 (CC-BY 4.0), que permite el uso, distribución y reproducción sin restricciones en cualquier medio, siempre que se acredite el autor y la fuente originales.

Consulte http://creativecommons.org/licenses/by/4.0/. ¿OPEN ACCESS

(c) (1) $(9)$

1 PhD. (C) Administración, Universidad Nacional de Colombia; Profesor, Escuela de Administración y Negocios - Facultad de Ciencias Económicas, Universidad de los Llanos; Grupo de Investigación Territorio y Ambiente; ORCID: 0000-0002-70985226; Autor de correspondencia: carosa@unillanos.edu.co.

\section{Resumen}

Las organizaciones campesinas, están inmersas en las actuales y complejas dinámicas de una sociedad de consumo, caracterizada por el oportunismo y agresividad, propias del capitalismo salvaje; visión que se debe aclarar es evidente en el mundo occidental. En este escenario, los trabajadores del campo como actores productivos están sujetos a exigencias que los obligan a adoptar cambios para poder permanecer en el mercado. Por otro lado, la situación de los agronegocios en una sociedad de consumo, sobre todo la de aquellos pequeños y medianos productores agrícolas, se caracteriza por su baja capacidad económica, por tanto, el acceso a la innovación tecnológica es limitado, causando de esta forma una brecha social y cultural, entre quienes pueden o no, acceder a la innovación.

Palabras Claves: Agronegocios, Cultivadores, Innovación, Consumo, Sociedad.

JEL: O32, Q13, F10

\section{Abstract}

The small farmer are immersed in the current and complex dynamics of a consumer society, which is characterized by opportunism and aggressiveness, typical of savage capitalism; vision that I must clarify, at least it is evident in the 
western world. In this scenario, farm workers as productive actors are subject to dynamics that force them to adopt changes in order to remain in the market. On the other hand, the situation of agribusiness in a consumer society, especially that of those small and medium farmers, is characterized by their low economic capacity, therefore access to technological innovation is limited, thus causing a gap social, among those who can or not, access to innovation.

Keywords: Agribusiness, Farmers, Innovation, Consumption, Society.

\section{Resumo}

Pequeno agricultor, estão imersas na atual e complexa dinâmica de uma sociedade de consumo, que se caracteriza pelo oportunismo e pela agressividade, típicos do capitalismo selvagem; visão que devo esclarecer, pelo menos é evidente no mundo ocidental. Nesse cenário, os trabalhadores agrícolas, como atores produtivos, estão sujeitos a dinâmicas que os obrigam a adotar mudanças para se manterem no mercado. Porém, a situação do agronegócio em uma sociedade de consumo, principalmente dos pequenos e médios agricultores, é caracterizada pela sua baixa capacidade econômica, pois o acesso à inovação tecnológica é limitado, causando assim um distanciamento social, entre quem pode ou não, acessar à inovação.

Palavras-chave: Agronegócio, Agricultores, Inovação, Consumo, Sociedade.

\section{Introducción}

El propósito del presente artículo de reflexión es el de discutir el rol del productor agrícola ante la innovación, en una sociedad de consumo que obliga a dinámicas de cambio constante, para tal fin, es necesario entender el contexto social y cul- tural en el que los agronegocios de producción agrícola se desarrollan. Entendiendo por agronegocios a aquellas unidades productivas que se dedican a cultivar la tierra y que se caracterizan en su mayoría por corresponder a organizaciones familiares e informales, como es el caso de los campesinos en los países en vía de desarrollo.

Los agronegocios tienen un peso significativo en el Producto Interno Bruto (PIB) en los países emergentes y por lo general gracias a ellos, se configuran sus sistemas agroalimentario y bioenergético, aspectos que son considerados por algunas naciones como un asunto de seguridad nacional. Estas organizaciones productivas desde una perspectiva holística, se revisten de gran complejidad, pues son concebidas como un conjunto de operaciones que hacen parte de una cadena de suministro cuyo valor está centrado en el reconocimiento que el consumidor pueda percibir en ellos (Clay \& Feeney, 2019), un consumidor que, por lo general, solo tiene contacto con el eslabón final, es decir, con el distribuidor y no con quien cultiva, a pesar de la importancia que éste desempeña en la entrega óptima del producto. Aunado a esto y por demás irónico, es el productor quien obtiene la menor participación en los márgenes de beneficio que cada eslabón gana por sus operaciones hacia el consumidor; incluso siendo el que asume el mayor riesgo.

Sumado a lo anterior, las condiciones de desigualdad que el productor agrícola enfrenta se agudizan en la sociedad moderna, dado los cambios en el comportamiento del consumidor en lo que respecta a sus gustos o preferencias agroalimentarias, obligando que los productores asuman mayores inversiones y riesgos para satisfacer las crecientes necesidades del mercado. Otro aspecto al que se enfrentan los productores agrícolas tradicionales, es el avance en las nuevas formas de producción, especialmente hacia los modelos industriales en 
las últimas décadas, que a través de organizaciones poderosas ejercen un control territorial generando imperfecciones de mercado que conllevan a fenómenos como el desplazamiento y la violencia en algunos casos (CEPAL, FAO, 2013; Valencia Toro y Mariño Arévalo, 2014).

\section{El rol de la innovación del productor agrícola en la sociedad liquida}

Los nuevos escenarios que brinda la sociedad de consumo, o en palabras de Bauman (2003), la sociedad del modernismo líquido, se caracteriza porque el cliente quiere estar más informado sobre la trazabilidad de lo que compra, exigiendo una mayor velocidad de los cambios en los productos; obligando de esta forma a que los productores tengan que adoptar innovaciones en los sistemas productivos. La implementación de estos cambios, que cada vez son más complejos a nivel tecnológico, genera dificultades para pequeños y medianos productores, ya que sugiere la adopción de innovaciones de tipo tecnológico en las prácticas agrícolas que por lo general son costosas o no son fáciles de adquirir, pero que son necesarias para alcanzar ventajas competitivas (Giacosa, Giachino, Bertoldi, \& Stupino, 2014; Jatib, 2003; Santos, Ferreira, De Araújo, De Oliveira, \& Clementino, 2017).

La situación anterior es descrita por la Organización de las Naciones Unidas para la Agricultura y la Alimentación (FAO), cuando menciona en su informe que el limitado acceso a la innovación es una de las más grandes problemáticas en el sector agrícola en el mundo (FAO, 2017)por parte de la Organización de las Naciones Unidas para la Alimentación y la Agricultura (FAO. De ahí la importancia de reflexionar sobre el rol del productor agrícola ante la innovación y las implicaciones culturales que el modernismo líquido implica para los trabajadores del campo.

\section{El rol del productor agrícola ante la innovación}

La baja innovación en los productores agrícolas no puede ser vista solo desde la falta de capacidad de un gobierno para brindar oportunidades a su sector productivo agrícola, también es importante considerar la cultura o comportamientos que poseen quienes lideran los agronegocios. En este sentido, los productores, es decir, los líderes de los agronegocios, quienes se caracterizan por poseer un estilo de liderazgo conservador, el cual constituye una barrera para la adopción de las innovaciones en el sector.

No se puede desconocer los beneficios que la innovación genera a las organizaciones, entre ellas las agrícolas, la importancia de identificar fuentes internas y externas que se constituyen en su capital relacional y la posibilidad de sostenerse en el mercado (Berdegué, 2005). Sin embargo, la innovación sugiere inversiones y diversos estados de relacionamiento de tal forma que el conocimiento compartido fluya hacia quien requiere innovar (Arosa-Carrera y Chica-Mesa, 2020), situación compleja en el sector agrícola dada la resistencia de éste hacia el cambio.

Estos procesos de resistencia a la innovación, presentado en la teoría de la destrucción creativa propuesta por Schumpeter (1997), conllevan a afirmar que el productor agrícola, quien funge como emprendedor (sujeto responsable de generar innovaciones), por lo menos en países en vía de desarrollo, no tendría las competencias necesarias para realizar los cambios, especialmente por su bajo nivel de cultura; además, de no contar con las herramientas para realizarlos de forma exitosa. En este sentido, el productor agrícola no generaría las oportunidades de desarrollo que le permitan romper el ciclo económico y tener mayor competitividad, lo que conlleva a 
una posición de inequidad en lo que respecta al poder de negociación en su cadena de suministro y como resultado una mayor desigualdad social para quienes trabajan el campo.

El dilema no es, si el productor debe cambiar o no, la sociedad de consumo le ha quitado esa posibilidad de decidir, éste entonces es, si las innovaciones que requiere el sector agrícola dependen de los elementos del contexto que el productor pueda obtener sin tener que hacer cambios culturales, o por el contrario, de su capacidad para cambiar comportamientos adoptando las innovaciones que exige una sociedad orientada al consumo, aquella que se caracteriza por imponer una rápida dinámica de cambios a los actores productivos. Esta postura en la que se coloca al productor agrícola es la que corresponde a procesos de innovación cuya relación se construye sólo si se asume el rol de emprendedor.

En este sentido, el rol del emprendedor ante la innovación puede ser de dos formas; el pasivo donde los productores agrícolas se vuelven sujeto de cambio de acuerdo con la dinámica que el mercado imponga, situación común en los agronegocios que lideran los pequeños y medianos productores. En contraste con ello, se encuentra el rol activo, que corresponde más a las grandes organizaciones de producción industrial, pues pueden realizar una innovación continua e inclusive destinar recursos con alto riesgo en procesos de innovación (Malerba \& Orsenigo, 1997); este último rol requiere de grandes inversiones en investigación y desarrollo, recursos con los que la mayoría de los agronegocios no cuentan, pues muchos de ellos no poseen los acervos, ni la capacidad para asumir el riesgo que supone invertir en estos conceptos.

La realidad del rol innovador en el campo por su escasa capacidad de invertir en investigación y desarrollo, los condena a ser actores pasivos y sujetos más de adopción que de creación de innovaciones, lo que en consecuencia crea una gran asimetría en lo que respecta al acceso a la innovación dentro del sector agrícola para poder cumplir de forma oportuna con los cambios del mercado.

\section{La sociedad del modernismo liquido y el productor agrícola}

Los pequeños y medianos agronegocios, de acuerdo con su rol pasivo, se limitan a realizar las innovaciones que el mercado les exige, con base en los intereses de quienes tienen el poder. La innovación en el productor, por consiguiente, está sujeta a los proveedores o clientes que conforman su cadena de suministro. Actores relacionales que están más informados de lo que sucede en el mercado al ejercer un poder discriminatorio sobre los agronegocios, al establecer de acuerdo con sus intereses comerciales, qué conocimiento compartirán con los agricultores (Rugeles et al., 2013).

En esta línea de análisis, el pequeño o mediano productor agrícola se considera un eslabón débil, que depende del actuar de proveedores e intermediarios, los cuales en ocasiones se comportan de forma oportunista (Calatrava, 2014). Aunque es obvio que toda la cadena de suministro debe cambiar de acuerdo con las dinámicas de consumo que la sociedad de la modernidad líquida impone, las dificultades para avanzar en los cambios se concentran con más fuerza en el productor; de forma irónica, es en la capacidad de innovar del productor donde toda la cadena cifra su éxito para cumplirle al mercado, pero el productor es quien obtiene menores ganancias de los márgenes de beneficios que cada uno de los eslabones de la cadena de suministro consigue del precio que el consumidor final paga.

En retoma del presente artículo se observa que el productor agrícola, en algunos casos llamado "campesino" de forma peyorativa por algunos 
miembros de las clases sociales mas favorecidas, alberga en el imaginario de la sociedad actual un estereotipo asociado a la pobreza, a las clases menos favorecidas y, por qué no decirlo, a la plebe. Estos comportamientos sociales desconocen el importante papel del productor agrícola en la sociedad, al punto de invisibilizarlo en los procesos de trazabilidad por parte del consumidor final, como si la salsa de tomate que está en la mesa viniera de la fábrica y no de una planta; o la leche que los consumidores toman todos los días no proviniese de la vaca, sino del refrigerador.

Sin embargo, el campesino, el líder, el emprendedor de un agronegocio de producción agrícola típico de los países no desarrollados, es quien debe adaptar su oferta a la sociedad, a pesar de experimentar discriminación por parte de ella, pues sus costumbres y hábitos son considerados de baja cultura. La sociedad entonces le exige innovaciones al productor agrícola con la finalidad de satisfacer cada vez más las cambiantes necesidades de consumo, esto por medio de la cadena de suministro de la que también hace parte, enviando mensajes de cambio a los productores, especialmente en exigencias técnicas, ambientales, sanitarias, entre otras.

Bajo este escenario, se configura la necesidad de generar acciones que permitan hacer del sector agrícola una actividad innovadora, donde se tendrá que decidir si el problema es el emprendedor —o sea, el campesino-, o el contexto. Si el problema es el primero, entonces el reto corresponde sobre todo a los ámbitos culturales, pues cambiar las costumbres y hábitos de los campesinos conllevará a procesos largos y dispendiosos. Por el contrario, si es el segundo, entonces se deberán proponer ambientes que faciliten el acceso del campesino a la innovación. Incluso podría pensarse en optar por una tercera vía y tratar de cambiar el contexto y al emprendedor al mismo tiempo. Claro, sin ser radical, pues de ahí podría pensarse que es más fácil remplazar el líder que formarlo, lo cual se traduce en políticas encaminadas a fomentar que los grandes cultivos industriales se impongan y que el campesino tradicional pase a ser una pieza más de los museos y parques temáticos, posesionándose de esta forma una nueva categoría de trabajador del campo.

En este último escenario, el nuevo y moderno trabajador dedicado al campo, no vendría acompañado de la nominación "campesino"; no podría decírsele a un cultivador culto, bien vestido e innovador, tal denominación. La palabra más adecuada para este actor de un mayor nivel sería entonces "Empresario del campo". Sin embargo, la discusión que plantea la reflexión no tiene que ver con la diferencia de clases, sino con la influencia que tiene la cultura en la concepción de quien lidera un agronegocio y el rol que éste ocupa en la sociedad de acuerdo con la dinámica social, es decir, aquella que parte de la interrelación entre cultura y economía, donde el productor agrícola es el eje del análisis.

A partir de las diferentes discusiones se plantea como supuesto: Qué los cambios exigidos al sector agrícola, por una sociedad de consumo, pueda terminar remplazando no a los actores sino a sus perfiles, es decir, no al productor, pero sí al campesino.

En esta instancia de la reflexión surgen dos preguntas: La primera ¿Cómo enfrentar a una sociedad de consumo que presiona la transformación del campesino o su extinción como lo conocemos? y la segunda ¿Es posible seguir conservando en el futuro el rol que actualmente conocemos del campesino, sin que esto sugiera su ruina en el sistema económico actual?; estas preguntas tienen que ver con las iniciativas que los investigadores de las ciencias de la gestión deben abordar sin perder de vista, la cultura y la seguridad ali- 
mentaria de una sociedad. Por ejemplo, el investigador puede entender que el primer interrogante presume el reconocimiento del campesino, como un miembro de la cultura que se debe conservar, pero cuyas condiciones culturales tradicionales hacia la innovación deben cambiar, pues una de las competencias de la sociedad del modernismo liquido, es que sea capaz de adoptar las innovaciones que el mercado impone de forma oportuna. Por otra parte, el desarrollar el segundo interrogante es aún más complejo, pues esto sugiere que los factores del entorno no son controlables y por ende los roles de los actores productivos, como es el caso del campesino, deben adaptarse a los cambios sociales e incluso en algunos aspectos debe poder diferenciarse de la competencia y ajustarse mejor al consumo, es decir el productor agrícola debe ser capaz de generar ventajas competitivas a costa de su esencia cultural.

Tal vez lo que estamos viendo en el sector agrícola no sea otra cosa que un proceso de construcción de una nueva cultura, la cual está creando diversas posibilidades en un universo social de relaciones entre consumidores finales y los trabajadores del campo. Una transformación que seguramente se acentuará más en las próximas décadas, cuando las nuevas generaciones, más formadas, vean en cultivar la tierra una oportunidad de negocio, en donde la condición sine qua non sea la innovación y donde el lenguaje sea otro.

Este otro lenguaje se nutrirá de forma dinámica, logrando así sincronizarse con la sociedad de consumo. Sin embargo, mientras esto se consolida, se deberá reconocer diferentes niveles de trabajadores del campo: en un extremo, ubicará a los nuevos y cultos empresarios, y en el otro a los campesinos, con el agravante de un contexto que genera más acceso a la innovación en el primer grupo y que limita de forma selectiva al segundo. Este argumento es coherente con la posición del profesor Licona, quien expresa que la cultura se configura como una supra categoría, creando jerarquías, en donde la hegemónica impone el lenguaje general y subordina a las demás (Licona, 2018).

Ahora bien, un reto a considerar es la gestión adecuada de un sistema agroalimentario que vive en constante enfrentamiento por los recursos de procedencia natural con un creciente sector bioenergético, lo cual podría ser beneficioso para el productor; sin embargo, la realidad es que los parámetros en técnicas de cultivo y especificaciones sanitarias, entre otros aspectos que se logran con la innovación tecnológica, hacen que para el pequeño y mediano empresario del campo, le sea difícil servir los mercados más interesantes y cuyo margen de beneficio son mejores, como el mercado de los bioenergéticos. Lo anterior hace que el campesino deba seguir en una cadena de suministro, donde es controlado tanto por los actores ascendentes como descendentes, cuya presión obliga a su transformación.

Es claro que el sistema agroalimentario no va a desaparecer, pero sí se va a sofisticar más, a modernizarse y darle paso a los cultivos industriales para consumo alimenticio y energético, liderados por un grupo de emprendedores denominado "empresarios del campo". quienes reciben su pago de grandes organizaciones que, con capacidad para generar una innovación permanente a través de sus modelos de gestión, dejarán cada vez más rezagados a los campesinos.

En este sentido, la interdisciplinaridad se impondrá en la gestión de la producción agrícola, las ciencias naturales y la ingeniería seguirán siendo útiles y continuarán como la base fundamental para la producción. Pero, a medida que el sector agrícola esté cada vez más condicionado a la sociedad de consumo, el nuevo emprendedor del campo deberá recurrir a áreas profesionales 
como la sociología y psicología, y a disciplinas como el marketing, entre otras, para poder comprender las necesidades y deseos de los consumidores y traducirlos en innovaciones tecnológicas relevantes que puedan ser adoptadas en sus agronegocios. Esto ocurre dada la complejidad que representa entender a la sociedad, la cual es imposible de abordar como fenómeno a partir de una sola disciplina específica (García, 2008).

\section{Conclusión}

La situación de los agronegocios en la sociedad del modernismo líquido parte de la imperiosa necesidad de generar capacidades en el productor agrícola que le permitan adoptar las innovaciones tecnológicas que las dinámicas del mercado actual imponen, cambios que cada vez son más frecuentes. Estas capacidades llevan a la transformación cultural del trabajador del campo, que poco a poco transita de campesino a empresario del campo, lo que da un nuevo lenguaje a las relaciones que el productor posee en la sociedad, y asimismo reconfigura la concepción cultural que tradicionalmente se tiene del sector agrícola.
En este sentido, la innovación se vuelve un requisito para la permanencia y sostenibilidad en el mercado de los agronegocios, ya que permite la creación de ventajas que no son otra cosa que el reconocimiento del valor por parte de los consumidores de acuerdo a sus necesidades y los nuevos deseos que la sociedad del modernismo liquido crea para satisfacerlos.

Por último, el nuevo papel del emprendedor del campo obliga a la transformación cultural, y poco a poco se irán tejiendo las nuevas relaciones y categorías de cultura entre los grupos sociales. Esta situación seguramente hará cambiar la actitud de las poblaciones urbanas hacia las rurales. Sin embargo, este no es un proceso que se vislumbre en el mediano plazo en todo el mundo, pues los procesos de cambios culturales que se impondrán en la nueva configuración del campo en países cuya economía todavía es precaria, ya se encuentran más avanzados en los países desarrollados, donde el área sembrada es mayor, las tecnologías están mas disponibles y los productores tienen una mayor educación. 


\section{Referencias}

Arosa-Carrera, C. R., \& Chica-Mesa, J. C. (2020). La innovación en el paradigma del marketing relacional. Estudios Gerenciales, 114-122. https://doi. org/10.18046/j.estger.2020.154.3494

Bauman, Z. (2003). Modernidad líquida (Fondo de C). México: Fondo de Cultura Económica.

Berdegué, J. (2005). Sistemas de innovación favorables a los pobres. Documento de antecedentes presentado al $29^{\circ}$. Periodo de sesiones del Consejo de Gobernadores del FIDA. Fondo Internacional de Desarrollo Agrícola (FIDA).

Calatrava, J. (2014). La innovación en el pensamiento económico consideraciones sobre su papel en el desarrollo endógeno de los territorios rurales y en el sector agroalimentario. Cuadernos de estudios agroalimentarios, 6, 27. Retrieved from http://www.publicacionescajamar.es / $\mathrm{pdf} /$ publicaciones-periodicas/cuadernos-de-estudios-agroalimentarioscea/6/6-672.pdf

CEPAL, FAO, I. (2013). Perspectivas de la agricultura y del desarrollo rural en las Américas: una mirada hacia América Latina y el Caribe: 2014. COEDICIONES. Retrieved from https://www.cepal.org/es/publicaciones/37136-perspectivas-la-agricultura-desarrollo-rural-americas-mirada-america-latina

Clay, P. Mac, \& Feeney, R. (2019). Analyzing agribusiness value chains: A literature review. International Food and Agribusiness Management Review. Wageningen Academic Publishers. https://doi. org/10.22434/IFAMR2018.0089

FAO. (2017). El estado mundial de la agricultura y la alimentación. Aprovechar los sitemas alimentarios para lograar una transformación rural inclusiva. https://doi.org/0251-1371

Garcia, R. (2008). Sistemas complejos. Conceptos, métodos y fundamentación epistemológica de la investigación interdisciplinaria. Barcelona: Gedisa editorial.

Giacosa, E., Giachino, C., Bertoldi, B., \& Stupino, M. (2014). Innovativeness of ceretto aziende vitivinicole: A first investigation into a wine company. International Food and Agribusiness Management Review, 17(4), 223-236.

Jatib, I. (2003). Food safety and quality assurance key drivers of competitiveness. International Food and Agribusiness Management Review, 6(1), 38-56.

Licona, W. (2018). Tensiones hermenéuticas en reflexiones de culturas, economía y lenguajes. In C. En Yáñez C (Ed.), Entre-Lugares de la Cultura (Editorial, pp. 215-239). Bogota, Colombia: Editorial Universidad Nacional de Colombia.

Malerba, F., \& Orsenigo, L. (1997). Technological Regimes and Sectoral Patterns of Innovative Activities. Industrial and Corporate Change, 6(1), 83-118. https:/ / doi.org/10.1093/icc/6.1.83 


\section{Referencias}

Rugeles, L., Guaitero, B., Saavedra, D., Betancur, I., Castillo, O., Arosa-Carrera, C., ... Vargas, M. (2013). Medición de la innovacón agropecuaria en Colombia (1st ed., Vol. 1). Medellin, Colombia: Sello Editorial Universidad de Medellin.

Santos, A. A. R., Ferreira, F. A., De Araújo, J. J., De Oliveira, D. G., \& Clementino, V. D. R. (2017). Dinâmicas de inovação: Análise das estratégias de inovação no cluster de manga da ride. Revista Em Agronegocio e Meio Ambiente, 10, 91114. https://doi.org/10.17765/21769168.2017v10nEd.esp.p91-114
Schumpeter, J. A. (1997). Teoría del desenvolvimiento económico : una investigación sobre ganancias, capital, interes y ciclo económico. Fondo de Cultura Económica. Retrieved from https://www.casadellibro.com/ libro-teoria-del-desenvolvimiento-economico-2-ed/9789681650698/820994

Valencia Toro, M., \& Mariño Arévalo, A. A. (2014). La empresa agroindustrial colombiana: un análisis de relaciones de poder y configuración de la apropiación de factores productivos. Equidad \& Desarrollo, 22(22), 79. https://doi. org/10.19052/ed.3251 